\title{
The Construction of Accurate Recommendation Model of Learning Resources of Knowledge Graph under Deep Learning
}

\author{
Xia Yang $\mathbb{D}^{1}$ and Leping Tan ${ }^{2}$ \\ ${ }^{1}$ Changsha Social Work College, Hunan, Changsha 410004, China \\ ${ }^{2}$ Hunan Vocational College of Science and Technology, Hunan, Changsha 410004, China
}

Correspondence should be addressed to Xia Yang; 20200090302048@email.sxu.edu.cn

Received 11 October 2021; Revised 30 October 2021; Accepted 10 November 2021; Published 25 January 2022

Academic Editor: Rahman Ali

Copyright (c) 2022 Xia Yang and Leping Tan. This is an open access article distributed under the Creative Commons Attribution License, which permits unrestricted use, distribution, and reproduction in any medium, provided the original work is properly cited.

\begin{abstract}
With the rapid development of science and technology and the continuous progress of teaching, it is now flooded with rich learning resources. Massive learning resources provide learners with a good learning foundation. At the same time, learners want to be precise from many learning resources. Second, it becomes more and more difficult to quickly obtain the learning resources you want. Therefore, it is very important to accurately and quickly recommend learning resources to learners. During the last two decades, a large number of different types of recommendation systems were adopted that present the users with contents of their choice, such as videos, products, and educational content recommendation systems. The knowledge graph has been fully applied in this process. The application of deep learning in the recommendation systems has further enhanced their performance. This article proposes a learning resource accurate recommendation model based on the knowledge graph under deep learning. We build a recommendation system based on deep learning that is comprised of a learner knowledge representation (KR) model and a learning resource KR model. Information such as learner's basic information, learning resource information, and other data is used by the recommendation engine to calculate the target learner's score based on the learner KR and the learning resource KR and generate a recommendation list for the target learner. We use mean absolute error (MAE) as the evaluation indicator. The experimental results show that the proposed recommendation system achieves better results as compared to the traditional systems.
\end{abstract}

\section{Introduction}

In recent years, the rapid development of artificial intelligence technologies such as deep learning, knowledge graphs, and artificial neural networks has driven society from "Internet + " into a new era of "artificial intelligence + ". The "Education Information 2.0 Action Plan" emphasizes on establishing and improving the sustainable development mechanism of educational information and building a networked, digital, intelligent, personalized, and lifelong education system [1]. The key to building a new education system lies in the development of personalized learning, and the realization of personalized learning is inseparable from the strong support of the adaptive learning system. The core components of the adaptive learning system include five parts: domain model, user model, adaptive model, adaptive engine, and presentation model [2]. The domain model is an important foundation and core element of building an adaptive learning system, and building a domain model with clear semantics, complete structure, and good scalability is an important challenge faced by adaptive learning systems. The artificial intelligence technology represented by the knowledge graph provides a technical guarantee for the construction of the educational field model. The "New Generation Artificial Intelligence [3] Development Plan" specifically pointed out that "focus on breakthroughs in core technologies of knowledge processing, in-depth search and visual interaction form a multi-source, multi-disciplinary and multi-data type cross-media knowledge map covering billions of entities." [4]. Therefore, the use of knowledge construction, knowledge mining, knowledge reasoning, and other technologies to realize the knowledge extraction, expression, fusion, reasoning, and utilization of the education domain model is an important topic in the current education 
research. The recommendation system is excellent in solving the "information overload problem." Traditional recommendation technologies, including collaborative filtering and content-based recommendations, have been applied in many fields, such as product recommendation, news recommendations, Amazon book recommendations, Netflix movie recommendations, and course recommendations $[5,6]$. In the e-learning environment, learners have different attributes, such as learning motivation, learning level, learning style, and preference, and these learner characteristics will affect the learner's learning. The general recommendation technology limits the performance of the online learning recommendation system due to cold start and sparsity issues [7-9]; that is, when there are new learning resources that have not been evaluated or new users who have not commented on any items, it will lead to the inability to make accurate recommendations. The data are huge, and the acquisition of these data will become very sparse [10]. Therefore, improving the accuracy of personalized learning resource recommendation and improving performance are currently an urgent problem to be solved. To solve the above problems, this paper mainly studies the recommendation algorithm based on knowledge representation and learning resources. To improve the customization and accuracy of recommendation, knowledge representation is used to integrate learners' learning level, learning style, and learning preferences into the recommendation process, and the semantic relationship of knowledge is used to alleviate the cold start problem; the problem of sparsity is solved through collaborative filtering algorithm.

The domain model construction refers to the process of using modeling technology to label and serialize domain knowledge. The education domain model construction refers to the process of using knowledge extraction, knowledge fusion, and other technologies to establish connections between subject knowledge and knowledge. The purpose is to serialize knowledge to better promote teaching and learning. The commonly used methods for model construction in the education domain are concept map, knowledge map, and knowledge graph. These three methods can perform knowledge representation and schemata, but these concepts are easy to confuse. The concept map was first proposed by Professor Novak to organize and characterize knowledge. It includes two parts, nodes and connections. Nodes are used to represent concepts, and connections are used to represent the relationship between concepts [6].

The concept map includes four elements: proposition, concept, cross-connection, and hierarchical structure. The concept map can not only describe knowledge but also evaluate knowledge. Trowbridge used concept maps to evaluate university courses and analyzed the differences in students' knowledge understanding of the same lesson; Ruiz-Primo used probability maps as an evaluation tool and found that the students' concept maps were interpreted as representing him. The concept of knowledge map was first proposed by Dansereau and Holley. The knowledge map can organize scattered knowledge to form serialized knowledge and promote the knowledge construction of learners. Knowledge map represents the path of knowledge acquisition and the relationship of knowledge. It can not only characterize the knowledge system structure, but also help learners accurately locate the required knowledge. The knowledge map is mainly used in the systematic construction of subject knowledge structure in the field of education. Kim believes that knowledge maps can establish knowledge relationships, help structure knowledge, and facilitate knowledge understanding; knowledge maps can help teachers reshape learning content and learning resources and effectively reflect the subject system, learning goals, and learning. At present, there are intelligent search engine, large-scale knowledge base, famous meta web knowledge base, wikista knowledge base of Wikimedia Foundation, and Yago comprehensive knowledge base developed by Max Planck Institute in Germany. In the field of education, Knewton in the United States used knowledge graphs to build an interdisciplinary knowledge system that includes concepts and their prerequisite relationships. Tsinghua University and Microsoft Research Institute developed Open Academic Graph, "Wisdom Learning Companion."

The main contributions of this research study include the following.

We build a recommendation system based on deep learning that is comprised of four parts: the learner knowledge representation (KR) model, the learning resource $\mathrm{KR}$ model, the CF recommendation engine, and the learning resource recommendation. These models include information such as the learner's basic information, the learning resource information, the data preprocessing modules, and the recommendation engine, which calculates the target learner's score based on the learner $\mathrm{KR}$ and the learning resource $\mathrm{KR}$ and generates a recommendation list for the target learner. We use mean absolute error (MAE) as the evaluation indicator to test the performance of our system and achieve a significantly lower MAE value as compared to the traditional CF algorithms.

The rest of the study is organized as follows.

Section 2 presents some of the related work by other researchers, Section 3 discusses the methodology of our approach, Section 4 discusses the experiments and their results, and Section 5 is the conclusion of our work.

\section{Related Work}

Liu Hongjing and others believe that the knowledge map can reflect the knowledge structure of a certain subject and promote learners to generate relevance and networked learning thinking. The knowledge graph was originally based on the measurement of scientific knowledge, showing the structural relationship of knowledge through graphical representation, which belongs to the research category of scientometrics. The knowledge graph is a structured knowledge semantic network. The nodes in the graph represent entities, and the edges in the graph represent the semantic relationships between nodes. Leo believes that the subject knowledge map, as a semantic network that establishes a connection between knowledge points and knowledge points, and knowledge points and teaching resources, 
TABLE 1: Comparison of concept maps, knowledge maps, and knowledge graphs.

\begin{tabular}{|c|c|c|c|}
\hline Dimension & Concept map & Knowledge map & Knowledge graph \\
\hline $\begin{array}{l}\text { Conceptual } \\
\text { connotation }\end{array}$ & $\begin{array}{l}\text { A tool for organizing and } \\
\text { characterizing knowledge }\end{array}$ & A structured knowledge network & $\begin{array}{l}\text { A semantic network that reveals the } \\
\text { relationships between entities }\end{array}$ \\
\hline $\begin{array}{l}\text { Constituent } \\
\text { elements }\end{array}$ & Nodes and connections & $\begin{array}{l}\text { Nodes, the relationship between nodes } \\
\text { and visual representation }\end{array}$ & Entity, attribute, semantic relationship \\
\hline $\begin{array}{l}\text { Range of } \\
\text { knowledge }\end{array}$ & For simple concepts & Target a clear learning topic & Wide range of domain knowledge \\
\hline $\begin{array}{l}\text { Knowledge } \\
\text { relations }\end{array}$ & $\begin{array}{l}\text { Relatively simple, often only } \\
\text { the whole and part of the } \\
\text { relationship }\end{array}$ & $\begin{array}{l}\text { The association relationship is relatively } \\
\text { rich, with parent-child, predecessor, } \\
\text { successor, inclusion, and other } \\
\text { relationships }\end{array}$ & $\begin{array}{c}\text { The knowledge relationship is a semantic } \\
\text { relationship, and the relationship is more } \\
\text { obtained through knowledge inference } \\
\text { mining }\end{array}$ \\
\hline $\begin{array}{l}\text { Application } \\
\text { field }\end{array}$ & $\begin{array}{l}\text { Knowledge representation, } \\
\text { knowledge organization, } \\
\text { knowledge evaluation }\end{array}$ & $\begin{array}{l}\text { Knowledge organization, knowledge } \\
\text { association, knowledge navigation, } \\
\text { knowledge search }\end{array}$ & $\begin{array}{l}\text { Intelligent search, in-depth question and } \\
\text { answer, social network, knowledge base } \\
\text { construction, knowledge reasoning }\end{array}$ \\
\hline
\end{tabular}

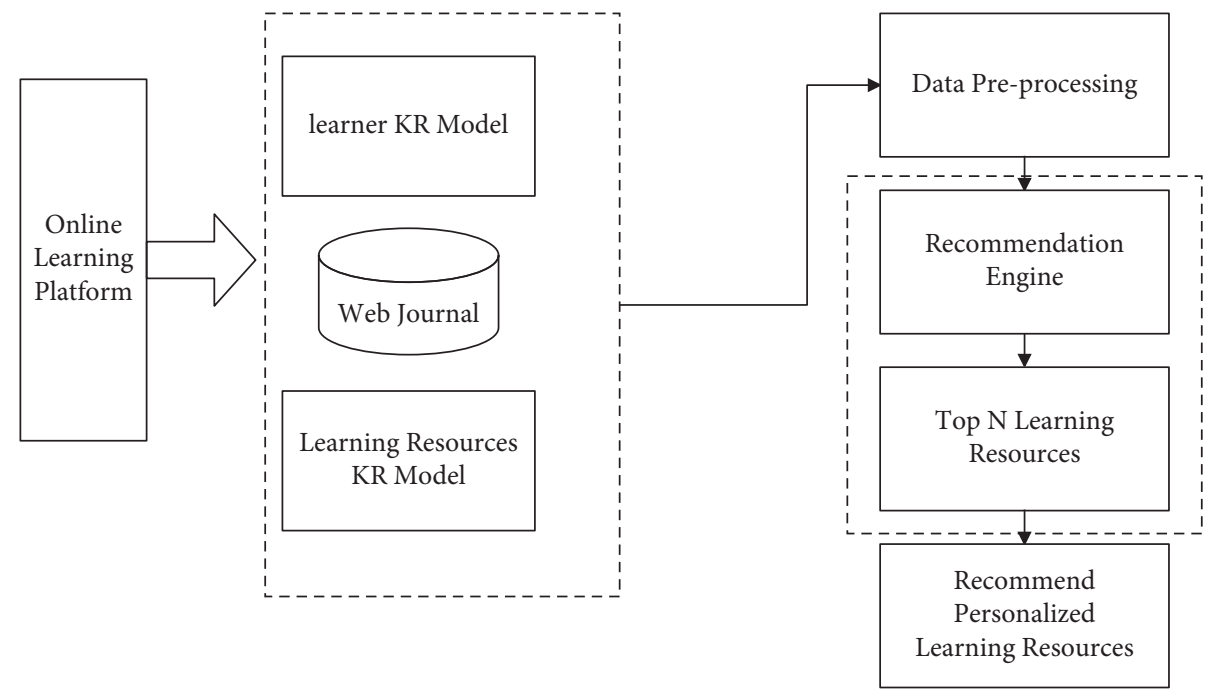

Figure 1: Accurate recommendation model of learning resources.

can play an important role in the semantic association of learning materials, the construction of learner models, and the personalized recommendation of learning resources; $\mathrm{Li}$ Zhen and others believe that the educational knowledge graph is a kind of knowledge element as a node, according to its multidimensional semantic relations to be associated, at the knowledge level and the cognitive level to represent the subject domain knowledge and the cognitive state of learners, available knowledge organization and cognitive representation tools for knowledge navigation, cognitive diagnosis, resource aggregation, and route recommendation; and $\mathrm{Yu}$ Shengquan and others believe that the subject knowledge graph is based on the logical relationship of knowledge formed by the semantic relationship of knowledge, on this basis, superimposes teaching goals, teaching problems, and cognitive status, and then generates a cognitive map. In the general field, knowledge graphs are mainly used in large-scale knowledge bases [11, 12]. Zhou et al. [13] incorporated word-oriented and entity-oriented knowledge graphs to enhance data representation in conversational recommendation systems and tackle the issue of insufficient contextual information and the issue of the semantic gap between natural language expression and user preference.
Shi et al. [14] proposed a knowledge graph-based learning path recommendation model to generate diverse learning paths and satisfy different learning needs. They build a multidimensional knowledge graph framework to store learning objects, proposed a number of semantic relationships between the learning objects, and built the learning path recommendation model. Zhou et al. [15] studied the various challenges the interactive recommender systems are facing, such as the large-scale data set requirement for the training of the model for effective recommendation. They suggested the use of knowledge graph for dealing with these issues and to use the prior knowledge learned from the knowledge graph to guide the candidate selection and propagate the users' preferences over the knowledge graph.

Based on the above analysis, this research conducted a comparative analysis of the three similar concepts of concept map, knowledge map, and knowledge graph from the five dimensions of conceptual connotation, component elements, knowledge scope, knowledge relationship, and application field and systematically sorted out the similarities of the three. The difference and the comparative analysis are shown in Table 1. To sum up, compared with concept maps and knowledge maps, knowledge graphs can express a wider 
range of knowledge content and semantic relations, and the construction is more automated. However, through literature analysis, it is found that the current knowledge graph still has the following problems in terms of knowledge content representation, learner ability description, and construction methods.

(1) In terms of knowledge content representation, the existing knowledge graph still focuses on the description of basic knowledge points. The knowledge content is scattered, and there is a lack of knowledge units that center on subject keywords and other related words are combined according to the semantic relevance of the subject knowledge cluster; (2) in terms of learner ability description, most of the existing knowledge graphs describe the content of knowledge points. There is a lack of further research on the relationship between knowledge points and the characterization of learners' abilities; and (3) in terms of construction methods, most of the existing knowledge graphs are still constructed manually by domain experts and lack the help of machine learning and automatic construction of artificial intelligence technologies such as deep learning and natural language processing.

\section{Method}

3.1. Accurate Recommendation Algorithm Based on Deep Learning. The attribute characteristics and learning resource characteristics of learners in the learning process are used as the basis for designing learner $\mathrm{KR}$ and learning resource KR models. On the basis of emphasizing learner's independent learning, based on CF and KR, we build an accurate recommendation model of learning resources as shown in Figure 1. The model mainly contains 4 parts: learner KR model, learning resource $\mathrm{KR}$ model, $\mathrm{CF}$ recommendation engine, and personalized learning resource recommendation. This article will explain in detail how the recommendation model works.

(1) Learner KR model: this model mainly includes learner's basic information, preference information, and learner attribute characteristic information. Learner's basic information can be obtained explicitly and implicitly; learner KR model stores learner attribute characteristics, including learning level, learning motivation, learning style, and other information. The learner KR model performs a personalized analysis of the learner's personal data according to the learner's preferences and attribute characteristics, while the CF recommendation engine uses the learner and the learning resource $\mathrm{KR}$ information to make score predictions for learning.

(2) Learning object model: this part stores the information of learning resources, including text, image, animation, audio or video, and other formats.

(3) Data preprocessing: the data preprocessing component is put in the learner, and learning resource data are prepared and preprocessed into the correct format that the recommendation engine can recognize.
(4) Recommendation engine: once the data preprocessing is successful, the recommendation engine will calculate the target learner score based on the learner and the learning resource KR, similarity, and the score prediction of the target learner. Finally, the recommendation engine generates a personalized recommendation list for the target learner.

3.1.1. Learners and Learning Resources. KR model is the organization method of subject knowledge and teaching laws. Its essence is the symbolic form of knowledge, mainly for the convenience of computers to store and process knowledge. At present, more KR technologies include predicate logic representation and Web representation, production rules, semantic network, and frame notation.

3.1.2. Learner KR Model. Since personalized learning resource recommendation needs to understand learner information and provides learning resources according to learners' learning goals, learning needs, teaching content, learning problems and environment, and preferences, it is necessary to establish a learner model. In the field of education, knowledge of learning resources, and concept retrieval, the KR-based learner model can feedback a series of concepts that are closely related to learning retrieval to learners based on the connections between concepts, which are conducive to the learners to discover new learning goals, areas of interest, and learning interests and make personalized recommendations. The formal definition of the learner model UR is as follows: $\mathrm{UR}=\left(U_{b}, U_{o}, U_{n}, U_{h}, U_{p}\right.$, $\left.U_{v}\right\}$, where the user's personal information $U_{b}=$ (ID, Name, Age, Sex, Edu, Tel), including basic personal information such as user name, name, age, education background, gender, and phone number. Learner $\mathrm{KR}$ prefers that the ontology is $U_{O}=\left(C, R_{C}, R_{N}, F_{C}, A, I\right)$, where $C$ represents the concept of learner preference in the ontology; RC describes the classification relationship between concepts in the learner's preference ontology; and RN describes the learner's preference ontology. FC represents the attribute and parity in the function. A means axiom; and Un is learning style information, and learning style is divided into 4 dimensions, active type/contemplative type, sentiment type/intuitive, visual/verbal, and comprehensive/sequential; that is, the learner's learning style is composed of 4 permutations and combinations; the different learning styles in this article are expressed as follows: $U_{\mathrm{n}}=$ (active/contemplative, perceptual/ intuitive, visual/verbal, comprehensive/sequential $)=\{1,2,3$, 4\}. $U_{\mathrm{h}}$ is the learning level information; to obtain the learning level of the learner, a random set of 10 questions is set for learning level test, through the test situation to assign the learning level of learners, where primary $=0 \sim 3$, intermediate $=4 \sim 6$, and advanced $=7 \sim 10$; for different learning levels, $U_{\mathrm{h}}$ is used to represent $U_{\mathrm{h}}=$ (primary, intermediate, advanced $)=\{1,2,3\}$. $U_{\mathrm{p}}$ is user preference information, which describes the user's preference for interface, font, language, resource content type, resource media type, etc. $U_{v}$ is access log information, which mainly records the time to 


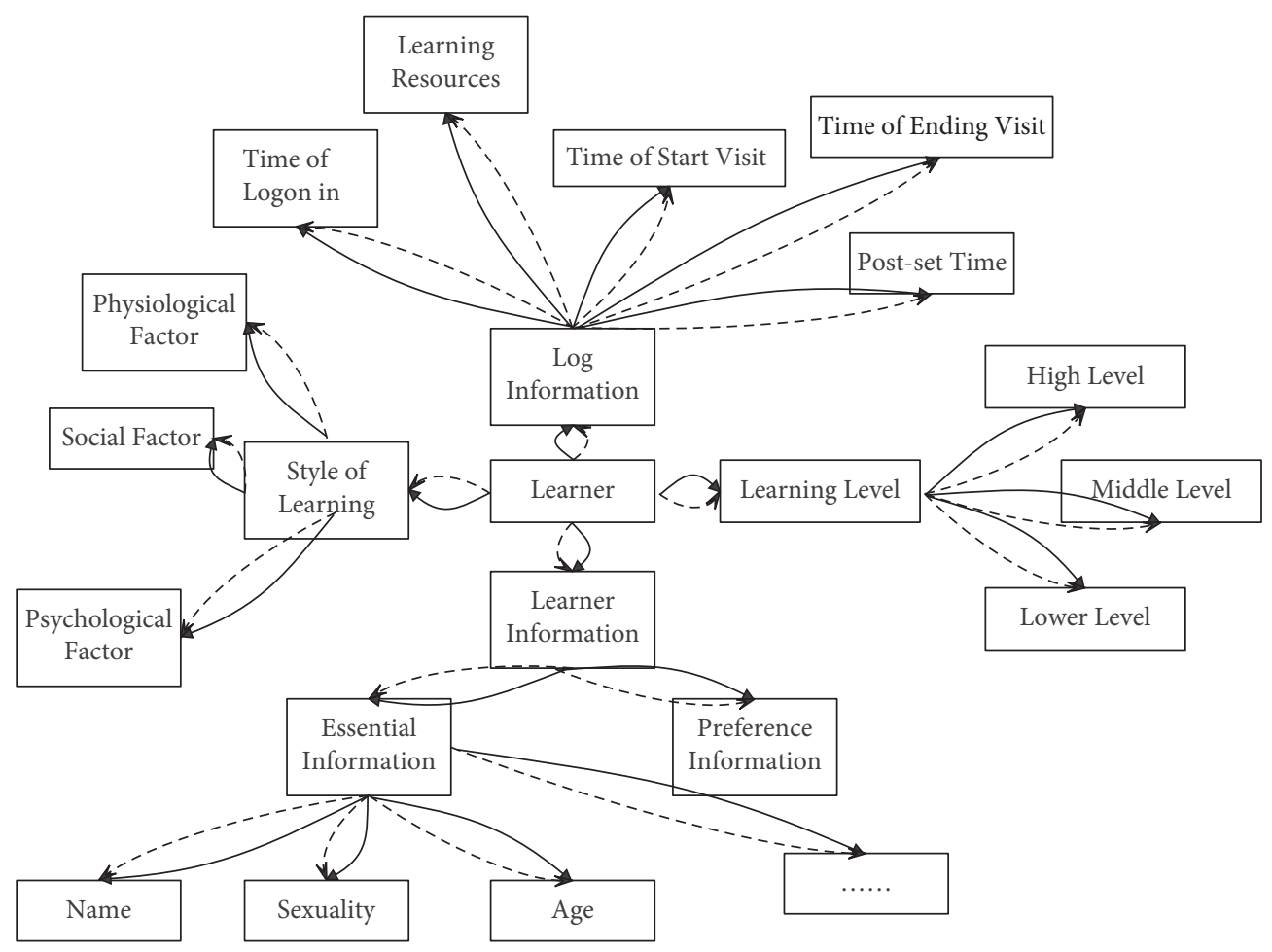

FIGURE 2: KR model of learner.

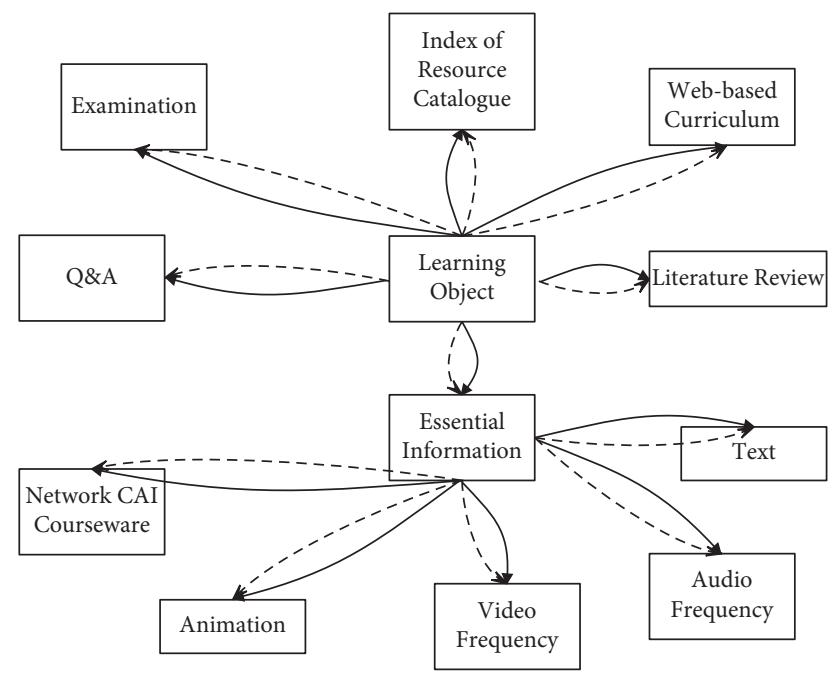

FIgURE 3: Model of learning resources.

log into the learning system, the learning resources accessed, the time to start accessing the learning resources, the time to complete the access, and the time to exit the system of learners, which can help them determine which time period is high in learning efficiency. Among them, the learner KR model is shown in Figure 2. The construction of the learner KR model needs to include the learner's personal data information, learning level, learning style information, and access log information and obtain the learner's data information through two methods, explicit and implicit. Moreover, it will learn learner profile information such as learner preferences, personal basic information (name, gender, age, etc.), learning style and learning level, and other attributes that are stored in the learner KR model. Once the learner's data information is obtained, the constructed learner model will be automatically updated to form personalized learner knowledge based on the learner's preferences, learning style, and learning level. The $\mathrm{CF}$ recommendation engine makes score predictions based on the learner's $\mathrm{KR}$ information and learning resource $\mathrm{KR}$ information, and the prediction score is high. The learning objects are recommended to the target learners, and then, personalized recommendations are made to the target learners. When a new learner enters the model, it will perform a semantic search based on the learner's registration information, analyze the learner's attribute characteristics, and determine the learner and match the learner's KR model to alleviate the cold start problem.

3.1.3. Learning Resource KR Model. In the online education environment, the structure of learning resources is complex and diverse. Its manifestations include courseware, cases, literature, resource catalog indexes, online courses, test questions, test papers, homework, text, animation, forum Q\&A, lesson plans, and media. To better build learning resources, data sharing is promoted between various types of learning resources at all levels, and the efficiency and accuracy of learning resource retrieval are improved; according to the characteristics of learning resource objects and the disorder of resource storage, the learning resources are divided into two categories, text materials and media materials. The text materials include courseware, cases, test 


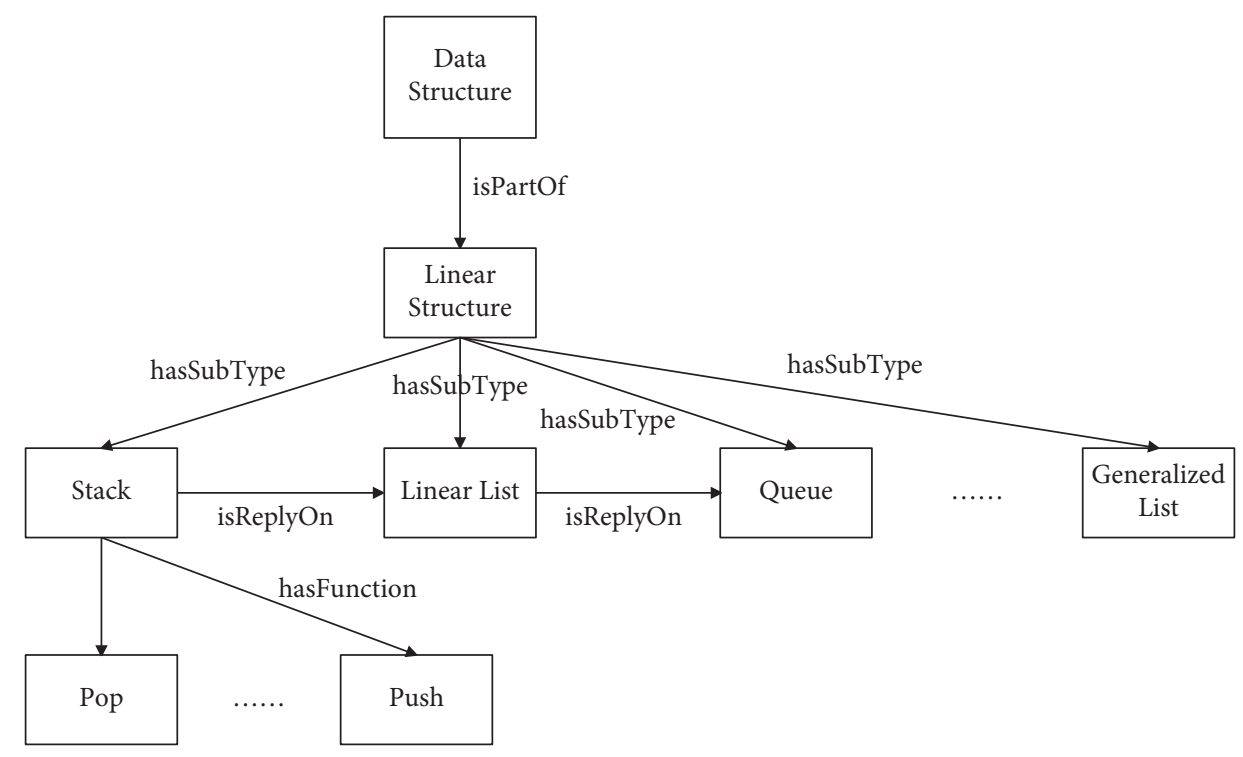

FIGURE 4: Hierarchy structure diagram of partial classes in "data structure."

papers, and textbook materials, while the media materials include graphics/image materials, animation materials, audio materials, and video materials. To be able to structure and describe learning resources, this article uses the metadata-based KR ontology construction method (metadatabased knowledge representation ontology building, referred to as MOB method) to define domain knowledge. This method is normative, having features such as rationality and scalability.

Firstly, the construction of the learning resource KR model can realize the management and retrieval of learning resources based on knowledge points. When constructing the learning resource description ontology, the ontology of computing subject knowledge points is used for representation, and the knowledge point ontology is mapped through the associated knowledge point attributes. Secondly, a list of terms is extracted from metadata: media materials, video/ audio, text, courseware, online courses, titles, resource locations, names, contact information, and association relationships, and finally, category level is defined and established. The class is defined according to the level of the classified resource. The learning object is the parent class (learning object), which includes subclasses such as courseware, text, and media data. The media data are text, video/audio, and graphics/pictures. Others are subcategories; the establishment of hierarchical relationships between categories mainly uses "part-of," "kind-of," "instanceof," and "attribute-of." The hierarchical structure diagram of the classes in the learning resource KR model is shown in Figure 3.

Constructing the learning resource KR model uses the ontology description language OWL and tools to express the relationship between the concepts and entities between learners, between learners and learning resources, and between knowledge points in learning resources. All the learning resources of CF are classified according to the attribute characteristics of each knowledge point, and examples and quizzes are carried out in the learning practice classroom. These quizzes and examples are closely related to the learning goals. The CF recommendation engine uses the semantic relationship between the learner and the learning resource. The learner performs score prediction and predicts the similarity of the target learner. Then, based on the data set provided by the online learning platform, the learner and learning resource $\mathrm{KR}$ model are constructed, and they are preprocessed together with the Web log data into CF82. The data structure course is taken as an example to apply it, mainly from the following aspects: (1) the key knowledge points of the data structure course; (2) the content related to the knowledge points, such as learning objectives, learning focus, and difficulty; (3) the relationship between the knowledge points before and after. The learning of the course is often contextual. Before learning a certain knowledge point, you must first learn another knowledge point; (4) examples explain the concept and use some examples or import before class to explain the concept, to help understand the concept. To be able to obtain the completeness of the resource more accurately, the class hierarchy is established using constraints and some special relationships between the classes. Standardized extraction is the hierarchical relationship between classes. Part of the class hierarchical structure constructed by taking the data structure as an example is shown in Figure 4.

\section{Experiment and Result Analysis}

The recommendation list of the CF recommendation system is generated in the following way. First, we find the nearest neighbors of several target learners, then extract the score data of the learning resources of the nearest neighbors, and finally predict the scores of the learning resources of the target learners based on these score data and generate recommendation list. The data set is extremely important for the algorithm. The data set is defined as data $=(U, I, R)$, where $U=\left\{u_{1}, u_{2}, u_{3}, \ldots, u_{m}\right\}$ is the basic learner set, $|U|=m$; 
$\mathrm{I}=\left\{i_{1}, i_{2}, i_{3}, \ldots, i_{n}\right\}$ is the collection of learning resources, $I \mid=n_{m} * n$-order matrix $R$ is the learner's scoring matrix for each learning resource, and the element rij indicates the score of the $i$ th user in $U$ on the $j$ th learning resource in $I$. The key to CF recommendation is to accurately locate the nearest neighbor of the target learner, and the basis for determining the nearest neighbor is to calculate the relationship between the learners. There are mainly the following three commonly used calculation methods for the similarity of equation (1).

4.1. Pearson's Correlation Similarity. The difference between the Pearson and the modified cosine similarity is that the denominator is the user's common rating item, shown as follows:

$$
\begin{aligned}
& S(u, v)=\frac{\sum_{\alpha \in P_{u v}}\left(R_{u, v}-\bar{R}_{u}\right)\left(R_{v, \alpha}-\bar{R}_{v}\right)}{\sqrt{\sum_{\alpha \in P_{u v}}\left(R_{u, \alpha}-\bar{R}_{u}\right)^{2}} \sqrt{\sum_{\alpha \in P_{u v}}\left(R_{v, \alpha}-\bar{R}_{v}\right)^{2}}}, \\
& S(u, v)=\frac{u * v}{u * v}
\end{aligned}
$$

The closer the value of $S(u, v)$ is to 1 , the higher the similarity between user $u$ and user $v$.

4.2. Adjusted Cosine. In view of the fact that the traditional cosine similarity does not consider the user's rating preference, that is to say, some users like high ratings and some like low ratings, for example, the physics and mathematics scores of two students are 5 and 4, 3 and 2, respectively. If the traditional cosine similarity calculation finds that the similarity between the two users is very low, but in fact the preferences of the two students are the same, compared with the mathematics class, two students prefer physics. The following equation is the modified cosine similarity of two users $u$ and $v$ :

$$
S(u, v)=\frac{\sum_{\alpha \in P_{u, \alpha}}\left(R_{u, \alpha}-\bar{R}_{u}\right)\left(R_{V, \alpha}-\bar{R}_{v}\right)}{\sqrt{\sum_{\alpha \in P_{u}}\left(R_{u, \alpha}-\bar{R}\right)^{2}} \sqrt{\sum_{\alpha \in P_{v}}\left(R_{v, \alpha}-\bar{R}_{v}\right)^{2}}} .
$$

Among them, $S(u, v)$ represents the set of scoring resource items, $P_{u}$ and $P_{v}$ represent the resource items scored by users $u$ and $v$, respectively, $R_{u, \alpha}$ and $R_{V, \alpha}$ represent the score of the resource item $\alpha$, and $R$ represents the average score. This article needs to consider the ontology domain. We use knowledge and users to evaluate learning resources. Therefore, in equation (4), we use the modified cosine similarity to calculate. The KR similarity calculation equation $S(i, j)$ of learning resource objects $i$ and $j$ is as follows:

$$
S(i, j)=\frac{\sum\left(R_{l, i}-\bar{R}_{l}\right)\left(R_{l, j}-\bar{R}_{l}\right)}{\sqrt{\sum\left(R_{l, i}-\bar{R}_{l}\right)^{2}} \sqrt{\sum\left(R_{l, j}-\bar{R}_{l}\right)^{2}}},
$$

where $R_{l, i}$ refers to the score of the learner $l$ on the learning resource object $i ; R_{l, j}$ refers to the score of the learner $l$ on the learning resource object $j$; and $R$ represents the average of all the scores provided by the learner $l$. Finally, the target learner's prediction score for the learning resource object is calculated. The $N$ most similar learning resource objects are obtained from equation (4). The calculation of the prediction score is shown in the following equation:

$$
P_{l, i}=\frac{\sum_{i \in N}\left(S(i, t) \times R_{l, t}\right)}{\sum_{i \in N} S(i, t)} .
$$

Among them, $N$ is a collection of learning resource objects like the learning resource object $i$, and $R_{l, t}$ is the scores of the learning resource object $t$ by the learner $l$.

The recommendation algorithm uses the $\mathrm{CF}$ recommendation engine to generate a top $\mathrm{N}$ recommendation list of learning resource objects based on the target learner's predicted scores of learning resources, the learner's knowledge representation, and the learning resource knowledge representation model. In this study, $L$ represents the set of all learners; that is, $L=\left\{l_{1}, l_{2}, l_{3}, \ldots, m_{n}\right\}$; I represents all possible recommended learning resources; that is, $I=\left\{i_{1}, i_{2}, i_{3}, \ldots, i_{n}\right\} ; k$ represents learners and learning resource domain knowledge; that is, $k=\left\{k_{1}, k_{2}, k_{3}, \ldots, k_{q}\right\}$; and $R$ represents the learner's scoring of learning resources, and the scoring range is defined as $R=\{1,2,3,4,5\}$.

Algorithm 1 generates a recommended list of top $\mathrm{N}$ learning resources. It is explained as follows.

This article applies the model to the self-developed "Mobile Autonomous School" online learning platform. To meet the individual learning needs of learners, starting from the two aspects of learners and learning resources, the learner KR model and learning resource KR are proposed. This model analyzes the learning behavior of students on the platform and comprehensively considers a personalized resource recommendation model based on the learner's learning level, learning style, and learning preferences. The model considers the learning characteristics and learning of the learners in actual learning applications and preference to introduce the best learning resources. It integrates the basic information of learners, learners' attributes, learners' hobbies, and user ratings to make the recommendation of learning resources more systematic and comprehensive. Among them, the recommendation of style information factors that consider learners' learning can enable learners to obtain recommended resources suitable for the learning situation at the time; the recommendation that takes into account the characteristics of the learner's attributes can enable the system to recommend learning resources that match the learner's learning level, academic background, and other characteristics; recommendations that consider learner preference factors can help promote learners' interest in learning recommended resources and improve the continuity of learning behavior; recommendations that consider learning goal factors make it easier for learners to learn efficiently and in a targeted manner; and the recommendation of evaluation factors can maintain the timeliness of recommended resources, so that learners can access the latest and most accurate learning resources. The personalized learning resource recommendation model combines the above attributes to systematically make 
Inputs:

collection of learning resource objects, $I=\left\{i_{1}, i_{2}, i_{3}, \ldots, i_{n}\right\}$.

$K R, k=\{$ learner, learning resource $\}$.

The score value of learner $R, R=\{1,2,3,4,5\}$.

Output:

prediction, score and top $\mathrm{N}$ learning resource recommendation list.

Method:

Step 1, fore-chain $\in \mathrm{I}, j \in \mathrm{I}, n \in \mathrm{N}$, do;

Step 2, Use equation (4) to calculate the similarity $S(i, j)$ End for each;

Step 3, Use formula (5) to calculate the prediction score $\mathrm{Pl}, i$;

Step 4, The top $\mathrm{N}$ learning resource objects with the highest predicted scores are used to generate a top $\mathrm{N}$ learning resource recommendation list for the target learner $l_{t}$.

Algorithm 1: Recommended list of top N learning resources.

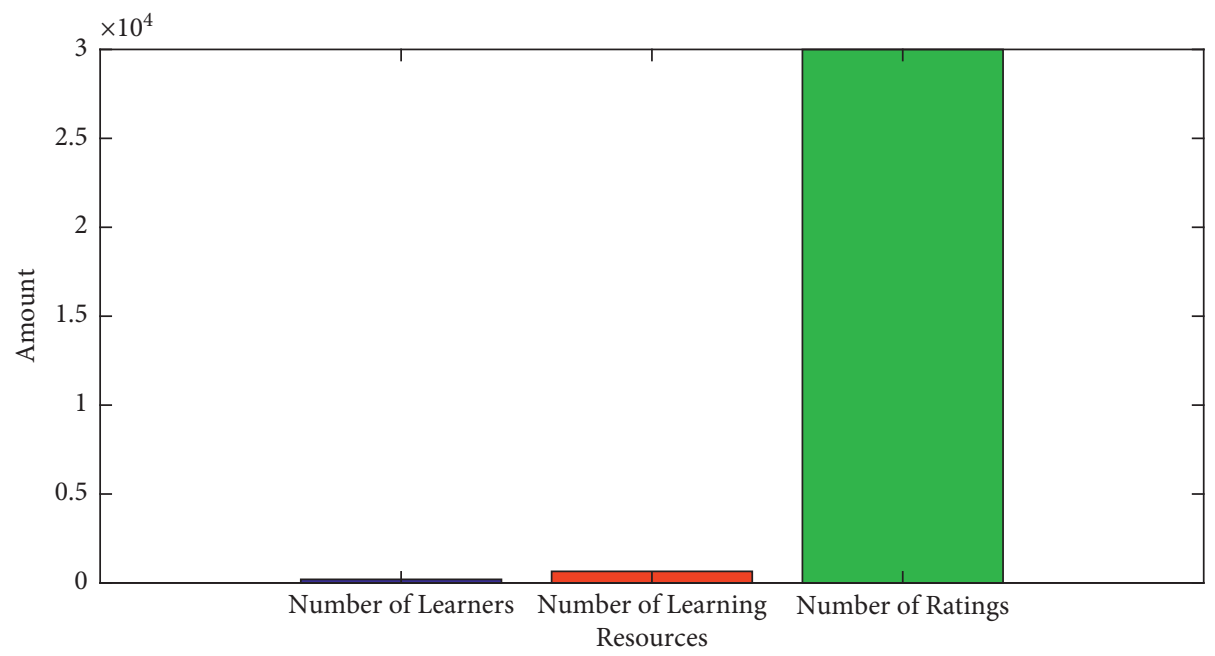

Figure 5: Data set.

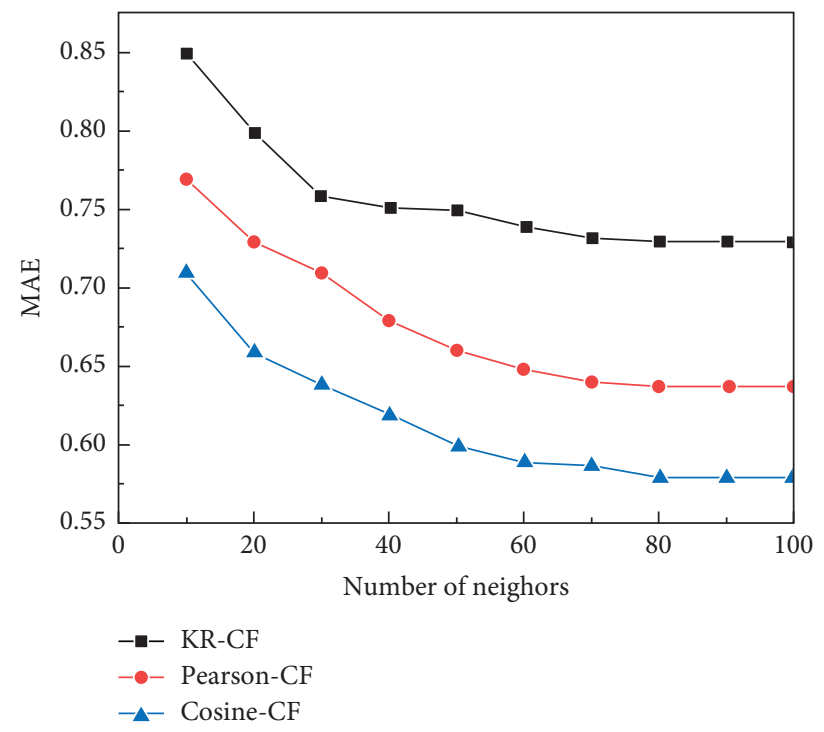

Figure 6: MAE values of the three algorithms. 


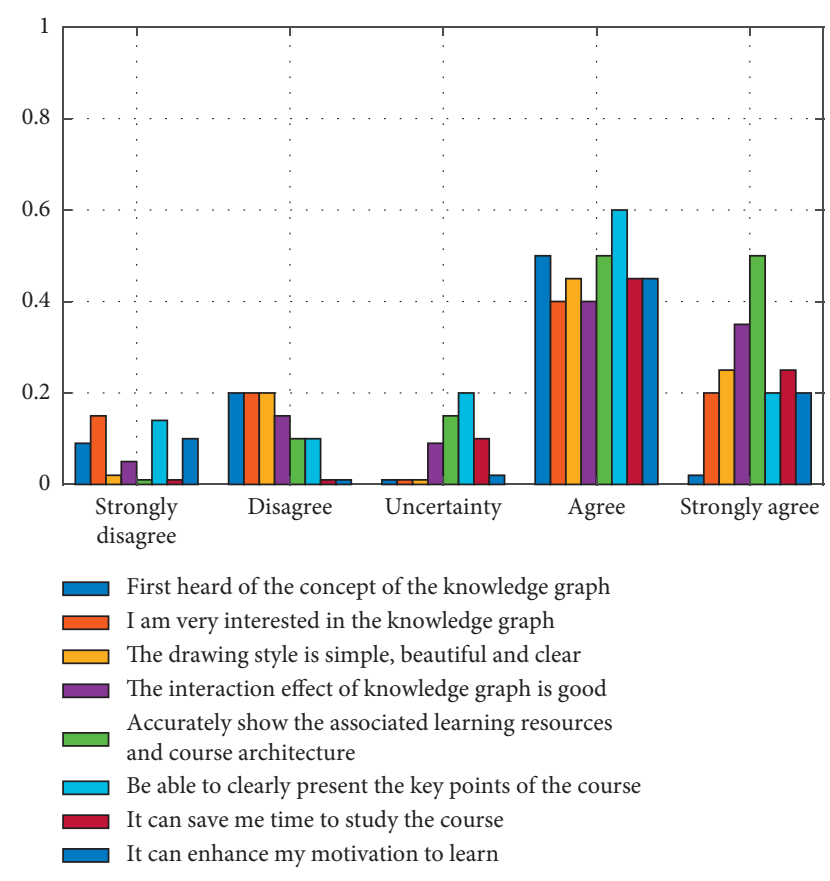

FIGURE 7: Effect of knowledge graph.

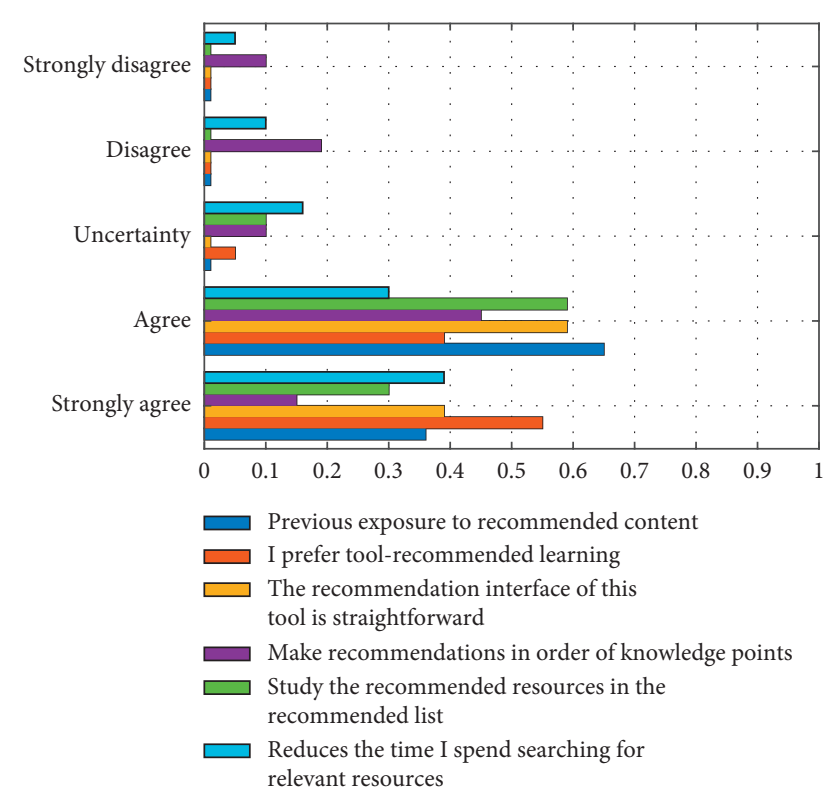

FIGURE 8: Digital resource recommendation effect.

personalized recommendations, saving learners. The time and energy of resources are chosen, and the utilization rate of learning resources and learners' academic performance is improved. The experimental data set contains 30,000 scoring records, generated from the evaluation and scoring of 650 learning resources by 200 learners within 2 months. The value range is $1 \sim 5$ (1-very irrelevant, 5 -very relevant), and 0 means that the learner has not made any evaluation. The experiment divides the collected data into two parts at a ratio of $1: 4$, and one part is used as the training set. The other part is used as a test set to

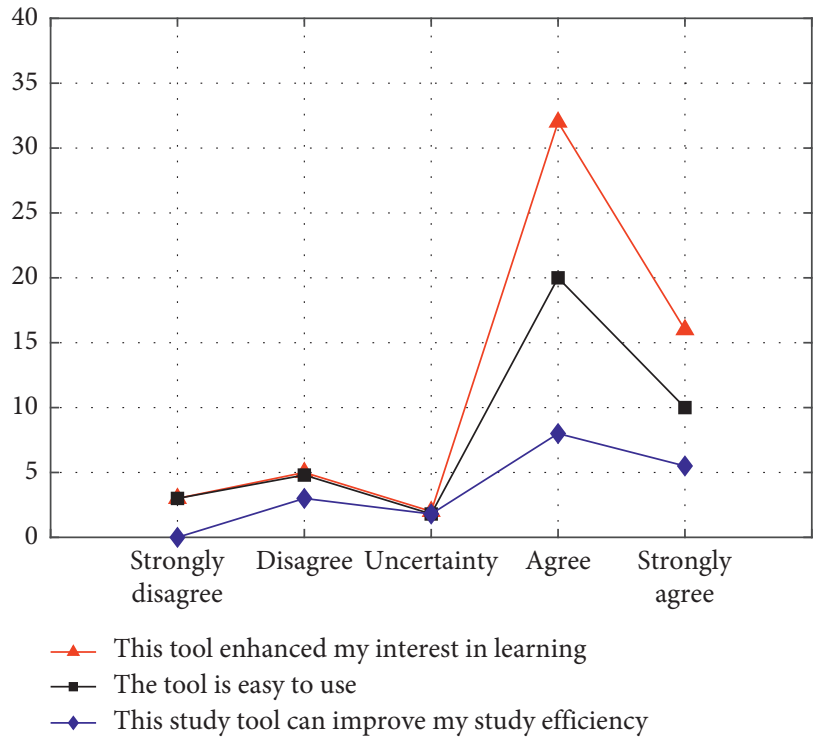

FIGURE 9: Effect of using the tool.

construct a recommendation model. The specific data are shown in Figure 5.

4.2.1. Algorithm Evaluation Criteria. This study uses the average absolute error (MAE) as an evaluation indicator to evaluate the accuracy of the proposed algorithm. MAE evaluates the performance of the recommendation system by accurately predicting the user's score [6]. MAE compares the user's project prediction score with the user's deviation of the actual score of the project, and the calculation formula for calculating the MAE of different neighbor communities through the algorithm proposed in this article is as follows:

$$
E^{\prime}=\frac{1}{m} \sum_{i=1}^{m}\left(P_{i}-R_{i}\right)
$$

Among them, $E$ is the average absolute error, $P_{\mathrm{i}}$ is the user's predicted score for the item, $R_{\mathrm{i}}$ is the user's actual score for the item, and $m$ is the number of predicted scores. From equation (6), the smaller the value of MAE, the higher the accuracy of the algorithm.

The KR-CF algorithm is compared with the traditional recommendation algorithms cosine-CF and Pearson-CF, and the comparison results are shown in Figure 6. From Figure 6, the MAE value of the KR-CF algorithm is significantly lower under a different number of neighbors. In traditional algorithms, as the number of neighbors increases, the sparseness of the data decreases; that is, the recommendation accuracy of the algorithm increases as the MAE value decreases and finally stabilizes. The KR-CF algorithm comprehensively considers learning when predicting the score. The learning level, learning style, and learning preferences of the learners improve the user similarity, which highlights the importance of the semantic relationship between learners for the recommendation of learning resources, improves the 
recognition accuracy of the learner's nearest neighbors, and makes the recommendation more reasonable. Experiments show that the KR-CF algorithm has a higher recommended performance than the traditional $\mathrm{CF}$ algorithm.

The questionnaire mainly investigates whether the user's usage habits of the tool and the resource recommendation of the knowledge graph are acceptable, to determine whether the tool can improve the learning efficiency of learners, reduce blindness in online learning, and achieve research purposes. The questionnaire's recognition of the learning effect of the recommendation tool is made into a Richter scale, which is considered from three aspects: the use effect of the curriculum resource knowledge map, the effect of digital resource recommendation, and the effect of comprehensive use of the tool. There are many settings in each aspect. We have questions for users to answer. A total of 20 questionnaires were distributed, and all were collected. According to the questionnaire, the histogram of the use effect of the curriculum resource knowledge map is as follows: the graph forms a normal distribution, and the agreement item generally becomes the highest value in this part, which shows that the use effect of the knowledge map is very satisfying, which is shown in Figure 7.

The line chart of the recommendation effect of digital resources shows that the abscissa represents the proportion of each item, and the ordinate represents the questions in the questionnaire. The experience of digital resources is good, and the percentage of agree and above is extremely high, which is shown in Figure 8.

From the perspective of the comprehensive use of the tool, this part examines the user's evaluation of the overall effect of the tool. The abscissa of the figure is the option, the ordinate represents the number of people, and the broken line represents the problem in this part. It can be seen from the line chart that most users agree that the tool is easy to use and can improve learning efficiency and enhance learning interest, which is shown in Figure 9.

Analyzing the three aspects of the questionnaire, the following results can be obtained:

(1) The interactive effect and user experience of the tool are good. $90 \%$ of the survey respondents agree with "the interactive effect of the knowledge graph is good," and "more than half of them agree very much." $80 \%$ of users have a simple, beautiful, and clear drawing style, and all users think that the recommended interface of this tool is simple and clear. Among them, $40 \%$ of users agree with this view very much.

(2) Very few people in the survey respondents have heard of the knowledge graph, but most people are very interested in it. In 20 learners, only $30 \%$ of the users have heard of the knowledge graph, and the remaining 70\% said that they once heard about the concept of knowledge graphs. Although most people are unfamiliar with knowledge graphs, $65 \%$ of users said they were "interested in knowledge graphs."
(3) The knowledge graph can present the structure of the course knowledge points and locate the key knowledge points. There are 14 users who agree with the "accurate display of related learning resources and course system structure," accounting for $70 \%$ of the total users, among which 4 people said they very much agree with this view. Using knowledge graphs, $85 \%$ of users agree that "the key knowledge points of the course can be clearly presented."

(4) In the process of online learning, resource recommendation is very necessary, and users are willing to learn the recommended content. The questionnaire shows that all surveyed users "have some contact with recommendation before," and 95\% of users "like resource tools." In the process of recommended learning, 11 of them agreed with this view. $60 \%$ of users agree that "tools can be recommended in the order of knowledge points in the course," and $80 \%$ of users are "willing to learn the recommended resource content in the recommendation list."

(5) The tool can save learning time and improve learning efficiency. The questionnaire shows that $65 \%$ of users believe that "it can save time for learning this course," and 14 people agree that "digital resource recommendation reduces the time to find related resources." The users who agree with "this learning tool can improve learning efficiency" account for $75 \%$ of the total number of users, indicating that most users believe that this tool can help improve learning efficiency.

\section{Conclusion}

This article intends to study the methods and applications of knowledge graphs in the recommendation of course digital resources. After doing a lot of literature research on knowledge graphs and recommendation techniques, the research background and related theories of knowledge graphs are analyzed, and the recommendation model based on knowledge graphs is summarized. Through the design and development of the digital resource recommendation tool, the role and significance of the knowledge graph in the field of digital learning resource recommendation are verified. As learning resources become more and more abundant, the recommendation of digital resources is bound to become the trend of adult learning and lifelong learning. Knowledge graphs play the most important role in this process. We elaborate the design ideas and function realization process of the resource recommendation tool based on the knowledge graph. Three modules are designed including the course digital resource uploading module, the course knowledge graph presentation module, and the related resource recommendation module. We implement a tool for recommending course digital resources based on knowledge graphs. Through specific course experiments, the usability and learning effect of this tool for adult online learning are analyzed. It is found by comparison that this tool can not only recommend relevant learning resources for 
learners, but also the interactive design on the knowledge map can stimulate learners' enthusiasm for online learning, enhance their interest in learning, and play a particularly important role in the effect of online learning. Experimental results show that the digital resource recommendation tool based on the knowledge graph meets the expected goals and design requirements, achieving a significantly lower MAE value as compared to its competitors. It also lays the foundation for the future research on resource recommendation based on the knowledge graph. The innovations of this research are mainly reflected in the method of presenting digital curriculum resources in the form of knowledge graph. This research proposes that the digital learning resources of curriculum are presented in the form of knowledge graph, which is different from the original linear knowledge point presentation method.

\section{Data Availability}

The data used to support the findings of this study are included within the article.

\section{Conflicts of Interest}

The authors declare that there are no conflicts of interest regarding the publication of this study.

\section{Acknowledgments}

This study was supported by the Hunan Provincial Natural Science Foundation Project: Construction of Accurate Learning Resource Recommendation Model Based on Knowledge Graphs (no. 2019JJ70062).

\section{References}

[1] M. Erdt, A. Fernandez, and C. Rensing, "Evaluating recommender systems for technology enhanced learning: a quantitative survey," IEEE Transactions on Learning Technologies, vol. 8, no. 4, pp. 326-344, 2015.

[2] J. Qiang, Research on the Support Model and Implementation Mechanism of Adaptive Learning System, Northeast Normal University, Changchun, 2012.

[3] X. Yang, T. T. Dong, and B. Ying, Web-based Adverse Event Reporting System for Hospital Nursing Care, Chinese Nursing Management, Beijing, China, 2012.

[4] The State Council of the People's Republic of China, New Generation Artificial Intelligence Development Plan, SCPRC, Zhongnanhai, Beijing, 2017.

[5] J. B. Schafer, F. Dan, J. Herlocker et al., Collaborative Filtering Recommend Systems, pp. 46-45, Spring, Berlin, 2007.

[6] M. K. Najafabadi and M. N. Mahrin, "A systematic literature review on the state of research and practice of collaborative filtering technique and implicit feedback," Artificial Intelligence Review, vol. 45, no. 2, pp. 1-35, 2015.

[7] HS. Le, "HU-FCF++: a novel hybrid method for the new user cold-start problem in commend systems," Engineering Applications of Artificial Intelligence, vol. 41, pp. 207-222, 2015.

[8] I. Barjasteh, R. Forsati, D. Ross, A.-H. Esfahanian, and H. Radha, "Cold-start recommendation with provable guarantees: a decoupled approach," IEEE Transactions on Knowledge and Data Engineering, vol. 28, no. 6, pp. 1462-1474, 2016.
[9] J. A. Konstan, J. D. Walker, D. C. Brooks et al., “Teaching commend system at large scale: evaluation and lessons learned from a hybrid MOOC[J]," ACM Transactions on ComputerHuman Interaction, vol. 22, no. 2, p. 10, 2015.

[10] S. Jie, Z. Jie, G. Yuan et al., "Natural language processing of nonstructural text of nursing adverse events and its effect[J]," Journal of ET Nursing, vol. 25, no. 3, pp. 1-4, 2018.

[11] J. Tarus, Z. Niu, and KhadidjaB, "E-learning recommender system based on collaborative filtering and ontology [J]. World academy of science, engineering and technology," International Journal of Computer, Electrical, Automation, Control and Information Engineering, vol. 11, no. 2, pp. 225-230, 2017

[12] J. Bobadilla, F. Ortega, A. Hernando, and A. Gutiérrez, "Recommender systems survey," Knowledge-Based Systems, vol. 46, no. 1, pp. 109-132, 2013.

[13] K. Zhou, W. X. Zhao, S. Bian, Y. Zhou, J. R. Wen, and J. Yu, "Improving conversational recommender systems via knowledge graph based semantic fusion," in Proceedings of the 26th ACM SIGKDD International Conference on Knowledge Discovery \& Data Mining, pp. 1006-1014, Newyark, USA, August 2020.

[14] D. Shi, T. Wang, H. Xing, and H. Xu, "A learning path recommendation model based on a multidimensional knowledge graph framework for e-learning," KnowledgeBased Systems, vol. 195, p. 105618, 2020.

[15] S. Zhou, X. Dai, H. Chen et al., "Interactive recommender system via knowledge graph-enhanced reinforcement learning," in Proceedings of the 43rd International ACM SIGIR Conference on Research and Development in Information Retrieval, pp. 179-188, Newyark, USA, July 2020. 